\title{
Strong and Electromagnetic Decays of the Baryon Decuplet
}

\author{
Malcolm N. Butler \\ Department of Physics, Stirling Hall \\ Queen's University \\ Kingston, Canada, K7L 3N6 \\ Martin J. Savage ${ }^{\dagger}$ \\ Department of Physics, 9500 Gilman Drive 0319 \\ University of California, San Diego \\ La Jolla, CA 92093-0319 \\ Roxanne P. Springer \\ Department of Physics \\ Duke University, Durham, NC 27706
}

\begin{abstract}
To explore further the role that QCD symmetries play on hadron dynamics, we discuss the radiative decay of the baryon decuplet to the baryon octet and compute the leading nonanalytic SU(3) violating corrections induced at the one loop level. In the limit of exact flavour SU(3) symmetry two of the possible decay modes are forbidden and the rates for these two decays are dominated by loop corrections. There is only one $\mathrm{SU}(3)$ conserving contact term at leading order in chiral perturbation theory for the radiative decays. We determine its coefficient from the observed branching ratio for $\Delta \rightarrow N \gamma$ and from the present upper limit on $\Xi^{* 0} \rightarrow \Xi^{0} \gamma$. We then predict the branching fractions for $\Sigma^{*-} \rightarrow \Sigma^{-} \gamma, \Sigma^{* 0} \rightarrow \Lambda \gamma, \Sigma^{* 0} \rightarrow \Sigma^{0} \gamma, \Sigma^{*+} \rightarrow \Sigma^{+} \gamma, \Xi^{* 0} \rightarrow \Xi^{0} \gamma$ and $\Xi^{*-} \rightarrow \Xi^{-} \gamma$. Some of the decay modes are predicted to have branching fractions near the current experimental upper limit and could possibly be observed in the near future. The leading corrections to the octet-decuplet-meson strong coupling constant, $\mathcal{C}$, are computed at one-loop and the strong decay rates of the $\Delta, \Sigma^{*}$ and $\Xi^{*}$ allow us to determine $|\mathcal{C}|=1.2 \pm 0.1$ and $\mathcal{H}=-2.2 \pm 0.6$, in remarkable agreement with predictions based on spin-flavour symmetry. We calculate the $\Delta$ pole graph contribution to the polarisability of the nucleon to order $1 / \Lambda_{\chi}^{2}$ in chiral perturbation theory.
\end{abstract}

UCSD/PTH 92-37, QUSTH-92-05, Duke-TH-92-44

November 1992

\footnotetext{
$\dagger$ SSC Fellow
} 


\section{Introduction}

The approximate $S U(3)_{L} \otimes S U(3)_{R}$ chiral symmetry of the strong interactions has recently been incorporated into a lagrangian that for the first time can consistently describe the dynamics of baryons near their mass-shell [1]. By performing a field redefinition, analogous to that used in heavy quark field theory (for a review see [2]), the large momentum associated with the classical trajectory of the baryon is removed, and one can write down a lagrangian that has consistent power counting in a loop expansion, and expansions in the baryon mass and chiral symmetry breaking scale. Calculations done using this lagrangian, therefore, probe the properties of QCD and help us understand how these manifest themselves in physical measurements. The energy range of validity for the method of heavy baryon chiral perturbation theory that will be used here encompasses many interactions that will be probed, for instance, at CEBAF.

In order to more deeply understand the significance of QCD symmetries, and their effects on baryon and meson processes, it is important to perform calculations on interactions that will be sensitive to these symmetries. One such involves the radiative decays from the baryon decuplet to the baryon octet. These are presented here. To understand the influence of meson loops on these decay rates, we study the SU(3) violating loop processes that probe them directly. Because the measurements of these radiative decay rates will be done soon, we believe it is pertinent to study them now, in a way that is model independent, and therefore more systematically accurate than attempts that have been made to date.

The strong decays of the baryon decuplet are calculated here to one loop. They also probe the underlying symmetries obeyed by baryons and mesons, and may lead to some (model independent) explanation of why the spin-flavor SU(6) symmetry, as implemented by the quark model, is so predictive.

\section{Heavy Baryon Chiral Perturbation Theory}

In this section we review the formalism of heavy baryon chiral perturbation theory (HBChPT) developed by Manohar and Jenkins [1]. The dynamics of the pseudogoldstone bosons arising from the breaking of $\mathrm{SU}(3)_{L} \otimes \mathrm{SU}(3)_{R}$ chiral symmetry to $S U(3)_{V}$ symmetry are described by the lagrangian

$$
\mathcal{L}_{M}=\frac{f_{\pi}^{2}}{8} \operatorname{Tr}\left[D^{\mu} \Sigma^{\dagger} D_{\mu} \Sigma\right]+\ldots . .
$$


With the normalizations used here, $f_{\pi} \approx 135 \mathrm{MeV}$ is the pion decay constant and $\Sigma$ is the exponential of the pseudogoldstone boson field,

$$
\Sigma=\exp \left(2 i M / f_{\pi}\right)
$$

where $M$ is the pseudogoldstone boson octet

$$
M=\left(\begin{array}{ccc}
\frac{1}{\sqrt{6}} \eta+\frac{1}{\sqrt{2}} \pi^{0} & \pi^{+} & K^{+} \\
\pi^{-} & \frac{1}{\sqrt{6}} \eta-\frac{1}{\sqrt{2}} \pi^{0} & K^{0} \\
K^{-} & \bar{K}^{0} & -\frac{2}{\sqrt{6}} \eta
\end{array}\right) .
$$

The covariant derivative includes the $U(1)_{Q}$ of electromagnetism

$$
D_{\mu}=\partial_{\mu}+i \mathcal{A}_{\mu}[Q,]
$$

where $\mathcal{A}$ is the electromagnetic field and $Q$ is the $\mathrm{SU}(3)$ charge operator.

The pseudogoldstone bosons have a well defined transformation under chiral symmetry, $\Sigma \rightarrow L \Sigma R^{\dagger}$, but a unique transformation does not exist for baryon fields. To introduce baryon fields consistently into the lagrangian, the field $\xi$ is defined, $\xi^{2}=\Sigma$, so that

$$
\xi=\exp \left(i M / f_{\pi}\right)
$$

This transforms under chiral symmetry as

$$
\xi \rightarrow L \xi U^{\dagger}=U \xi R^{\dagger}
$$

where $U$ is defined implicitly by (2.6). From this, vector fields with definite parity can be defined:

$$
V^{\mu}=\frac{1}{2}\left(\xi D^{\mu} \xi^{\dagger}+\xi^{\dagger} D^{\mu} \xi\right), \quad A^{\mu}=\frac{i}{2}\left(\xi D^{\mu} \xi^{\dagger}-\xi^{\dagger} D^{\mu} \xi\right)
$$

where $A_{\mu}$ transforms homogeneously and $V_{\mu}$ transforms inhomogeneously under chiral symmetry.

The work of ref. [1] shows that processes with baryons that are nearly on their mass shell can be consistently described by a chiral lagrangian involving fields of definite velocity. In this representation the chiral lagrangian becomes an expansion involving both $k / \Lambda_{\chi}$ and $k / M_{B}$, where $\Lambda_{\chi}$ is the chiral symmetry breaking scale (of order $1 \mathrm{GeV}$ ), $k$ is the residual off-shellness of the nucleon, and $M_{B}$ is the mass of the baryon. These expansion parameters are both much less than unity, as required for such an expansion to be useful in calculations. 
In contrast, an expansion in $P / \Lambda_{\chi}$ (of order unity) where $P$ is the full momentum of the baryon, and each order in the derivative expansion is equally important, does not satisfy this requirement. The HBChPT allows for a systematic expansion in powers of $1 / M_{B}$ and $1 / \Lambda_{\chi}$ (for a review see [3]). The lagrangian describing the strong interactions of the lowest lying octet of baryons with the pseudogoldstone bosons to lowest order in $1 / M_{B}$, lowest order in derivatives, and lowest order in the quark mass matrix is

$$
\mathcal{L}_{v}^{8}=i \operatorname{Tr} \bar{B}_{v}(v \cdot \mathcal{D}) B_{v}+2 D \operatorname{Tr} \bar{B}_{v} S_{v}^{\mu}\left\{A_{\mu}, B_{v}\right\}+2 F \operatorname{Tr} B_{v} S_{v}^{\mu}\left[A_{\mu}, B_{v}\right]
$$

where

$$
B_{v}=\left(\begin{array}{ccc}
\frac{1}{\sqrt{2}} \Sigma_{v}^{0}+\frac{1}{\sqrt{6}} \Lambda_{v} & \Sigma_{v}^{+} & p_{v} \\
\Sigma_{v}^{-} & -\frac{1}{\sqrt{2}} \Sigma_{v}^{0}+\frac{1}{\sqrt{6}} \Lambda_{v} & n_{v} \\
\Xi_{v}^{-} & \Xi_{v}^{0} & -\frac{2}{\sqrt{6}} \Lambda_{v}
\end{array}\right)
$$

and the covariant chiral derivative is $\mathcal{D}_{\mu}=\partial_{\mu}+\left[V_{\mu}, \quad\right]$. The subscript $v$ indicates that the fields are redefined to remove baryon mass factors, and are of definite velocity. Therefore, only factors of the residual off-shell momentum are generated by derivatives acting on the baryon field. The spin operator, $S_{v}$, acts on the baryon fields. The strong interaction coupling constants $F$ and $D$ have been determined at the one-loop level in chiral perturbation theory [1] to be $F=0.40 \pm 0.03$ and $D=0.61 \pm 0.04$. The strong interactions of the lowest lying decuplet of baryon resonances are incorporated to the same order in $1 / M_{B}$, derivatives, and the quark mass matrix by

$$
\begin{aligned}
\mathcal{L}_{v}^{10} & =-i \bar{T}_{v}^{\mu}(v \cdot \mathcal{D}) T_{v \mu}+\Delta m \bar{T}_{v}^{\mu} T_{v \mu}+\mathcal{C}\left(\bar{T}_{v}^{\mu} A_{\mu} B_{v}+\bar{B}_{v} A_{\mu} T_{v}^{\mu}\right) \\
& +2 \mathcal{H} \bar{T}_{v}^{\mu} S_{v \nu} A^{\nu} T_{v \mu}
\end{aligned}
$$

where the elements of $T_{v}$ are (suppressing the Lorentz index and velocity subscript)

$$
\begin{aligned}
& T^{111}=\Delta^{++}, \quad T^{112}=\frac{1}{\sqrt{3}} \Delta^{+}, \quad T^{122}=\frac{1}{\sqrt{3}} \Delta^{0}, \quad T^{222}=\Delta^{-}, \quad T^{113}=\frac{1}{\sqrt{3}} \Sigma^{*+} \\
& T^{123}=\frac{1}{\sqrt{6}} \Sigma^{* 0}, \quad T^{223}=\frac{1}{\sqrt{3}} \Sigma^{*-}, \quad T^{133}=\frac{1}{\sqrt{3}} \Xi^{* 0}, \quad T^{233}=\frac{1}{\sqrt{3}} \Xi^{*-}, \quad T^{333}=\Omega^{-}
\end{aligned} .
$$

The mass splitting between the decuplet of resonances and the octet of baryons is $\Delta m$. The coupling constant $\mathcal{H}$ cannot be extracted directly from experimental data but because the decuplet baryons appear in loop processes it can be extracted indirectly. The coupling constant, $\mathcal{C}$, can be found from the strong decay of decuplet baryons to octet baryons. 


\section{Strong Decays of the Decuplet}

The strong decay of the decuplet of baryon resonances to the baryon octet is determined by the coupling constant $\mathcal{C}$ of $(2.10)$. Experimentally, the values of $\mathcal{C}$ extracted from the decay rates of the processes $\Delta \rightarrow N \pi, \Sigma^{*} \rightarrow \Sigma \pi, \Sigma^{*} \rightarrow \Lambda \pi$, and $\Xi^{*} \rightarrow \Xi \pi$ are centered about 1.5, 1.4,1.4, and 1.3, respectively. These, as expected, have SU(3) violation at the $30 \%$ level. The tree level amplitude for these processes is $\mathrm{SU}(3)$ conserving, which prompts us to look at loop corrections. The leading SU(3) violation to these decays arises from the graphs shown in fig. 11. We retain the leading nonanalytic terms of the form $m_{K}^{2} \log \left(m_{K}^{2} / \mu^{2}\right)$, choosing the renormalisation point $\mu$ to be the chiral symmetry breaking scale $\Lambda_{\chi}$. We include the contribution of graphs involving $\eta$ mesons, by relating them to those involving $K$ mesons, through the Gell-Mann-Okubo mass formula $m_{\eta}^{2}=\frac{4}{3} m_{K}^{2}$. The corrections to this are of order $m_{q} / \Lambda_{\chi}$, where $m_{q}$ is the current quark mass.

We find that with the nonanalytic contributions, the relevant vertex at one-loop is

$$
\mathcal{C}\left(1-\left(\alpha_{T B}+\bar{\lambda}_{T B}\right) \frac{m_{K}^{2}}{16 \pi^{2} f_{K}^{2}} \log \left(m_{K}^{2} / \Lambda_{\chi}^{2}\right)\right)\left(\bar{T}^{\nu} A_{\nu} B+\bar{B} A_{\nu} T^{\nu}\right)
$$

where $T$ is a baryon resonance in the decuplet, $B$ is an octet baryon, $A_{v}$ is the axial field defined earlier, and $f_{K}$ is the kaon decay constant. The coefficient $\alpha_{T B}$ comes from the one-particle irreducible graphs shown in fig. 1, and $\bar{\lambda}_{T B}=\frac{1}{2}\left(\lambda_{T}+\lambda_{B}\right)$ is the contribution from the wavefunction renormalisation graphs shown in fig. 2. The expressions for $\alpha_{T B}$ and $\lambda_{T B}$ are given in Appendix A. We use the values of $F=0.40$ and $D=0.61$ extracted at one-loop from axial current matrix elements[1], and fit $\mathcal{C}$ and $\mathcal{H}$ to the four decays: $\Delta^{++} \rightarrow p \pi^{+}, \Sigma^{*+} \rightarrow \Sigma^{0} \pi^{+}, \Sigma^{*+} \rightarrow \Lambda \pi^{+}$, and $\Xi^{* 0} \rightarrow \Xi^{-} \pi^{+}$. Plotted in fig. 3 is $\mathcal{C}$ as a function of $\mathcal{H}$ for each decay mode. The four rates can be consistently described in terms of one value for the coupling constant $\mathcal{C}$ if $1.1<|\mathcal{C}|<1.3$. This requires that the coupling constant $\mathcal{H}$ is constrained to lie in the interval $-2.8<\mathcal{H}<-1$.6. This is consistent with the constraint found in ref. [1] of $\mathcal{H}=-1.9 \pm 1.0$. These two determinations are independent in the sense that they arise from experimental data on completely different processes. So we have some indication that our computation of the allowed region for $\mathcal{H}$ is valid.

The above results impact discussions on the spin-flavor SU(6) symmetry. The heavy baryon octet and decuplet fields may be combined into a single $\mathbf{5 6}$ representation of an $\mathrm{SU}(6)$ spin-flavour group [1] [3] 团. The predictions arising from the application of this symmetry work well for many interactions, despite the fact that from a QCD standpoint this symmetry should be completely destroyed by the strong dynamics. If it is assumed 
that the goldstone boson field, $A_{\mu}$, transforms as a 35 of SU(6) (instead of a 405 or 2695), then the following relations emerge between coupling constants (the same relations can be found from non-relativistic quark models):

$$
\begin{aligned}
& F=\frac{2}{3} D \quad \mathcal{C}=-2 D \\
& \mathcal{H}=-3 D
\end{aligned}
$$

The computations performed in ref. [1] show that the first relation, $F=\frac{2}{3} D$, is very well satisfied in HBChPT. We cannot extract the sign of $\mathcal{C}$ (it has no physical consequence in HBChPT), but find the relation, $|\mathcal{C}|=2 D$, is also very well satisfied (with the central values of $|\mathcal{C}|=1.2$ and $2 D=1.2$.) The third relation, $\mathcal{H}=-3 D$, would indicate that $\mathcal{H} \sim-1.8$. This is within the $1 \sigma$ region extracted for $\mathcal{H}$ from fig. 3. It seems that the relations between coupling constants that come from invoking an $\mathrm{SU}(6)$ spin-flavour symmetry are all well satisfied in HBChPT calculations. This lends credence to the idea, discussed in ref.[3], that there may well be an underlying $\mathrm{SU}(6)$ symmetry manifest in the hadronic sector.

\section{Radiative Decays of the Decuplet}

Radiative decays of the decuplet of baryon resonances provide insight into the electromagnetic interaction of baryons complementary to that obtained from static properties of the lowest lying baryon octet. The Coleman-Glashow relations for the magnetic moments of octet baryons [5] in the limit of exact SU(3) symmetry work remarkably well and require that loop-induced $\mathrm{SU}(3)$ violation be small [6] [7]. In contrast, little is known about the radiative decays of the decuplet of baryon resonances. The branching ratio for the radiative decay of the $\Delta$ is inferred to be $0.0061 \pm 0.0005$ [8], while only upper limits exist for the branching ratios of the $\Sigma^{*}$ and $\Xi^{*}$ radiative decays. Meanwhile, the theoretical investigations have been limited to relativistic quark models and nonrelativistic potential models [9], where it was found that the decay rates were very sensitive to the details of the model (Primakoff production of the resonances via hyperon beams has also been examined in the context of the quark model [10]). We will use chiral perturbation theory to make predictions for the radiative decay modes of the decuplet of baryon resonances.

There is one SU(3) conserving, dimension five, counterterm that contributes to the radiative decay of the baryon decuplet of the form

$$
\mathcal{L}_{v}^{T B \gamma}=i \Theta \frac{e}{\Lambda_{\chi}} \bar{B}_{v} S_{v}^{\mu} Q T_{v}^{\nu} F_{\mu \nu}
$$


where $Q$ is the electromagnetic charge matrix,

$$
Q=\left(\begin{array}{ccc}
2 / 3 & 0 & 0 \\
0 & -1 / 3 & 0 \\
0 & 0 & -1 / 3
\end{array}\right)
$$

and $\Theta$ is an unknown coefficient that we will subsequently determine. Note that, formally, there is an analogous counterterm proportional $1 / M_{B}$. Here, this type of term is absorbed into the above operator. In the limit of exact $\mathrm{SU}(3)$, this term forbids the decays $\Sigma^{*-} \rightarrow$ $\Sigma^{-} \gamma$ and $\Xi^{*-} \rightarrow \Xi^{-} \gamma$. This is a consequence of the $V$-spin invariance of both the strong and electromagnetic interactions. However, these decays are induced at loop level through the $\mathrm{SU}(3)$ violation that occurs from keeping explicit the $K$ and $\pi$ masses in the loop diagrams, and by insertions of the quark mass matrix in local operators.

At one-loop, two graphs contribute to the radiative decay of decuplet baryons. These are shown in fig. 4 . It is these graphs that give the leading $\mathrm{SU}(3)$ violating terms in the decay rates, and are therefore the dominant contribution to the decays $\Sigma^{*-} \rightarrow \Sigma^{-} \gamma$ and $\Xi^{*-} \rightarrow \Xi^{-} \gamma$. In calculating the two graphs in $4-2 \epsilon$ dimensions, it is convenient to define

$$
\begin{aligned}
& I_{1}=\int_{0}^{1} d x x \Gamma(\epsilon) I\left(-\epsilon, \omega_{\gamma} x-\Delta m, M^{2}\right) \\
& I_{2}=\int_{0}^{1} d x(x-1) \Gamma(\epsilon) I\left(-\epsilon, \omega_{\gamma} x-\Delta m, M^{2}\right)
\end{aligned}
$$

where $\omega_{\gamma}$ is the energy of the photon emitted during the decay, $\Delta m$ is the mass difference between the initial decuplet baryon and the intermediate state baryon, $M$ is the mass of the goldstone boson in the loop, and

$$
I(a, b, c)=\int_{0}^{\infty} d \lambda\left(\lambda^{2}+2 b \lambda+c\right)^{a}
$$

Recursion relations and rules for the latter integral are discussed in ref. [3]. An SU(3) symmetric $\overline{\mathrm{MS}}$ subtraction scheme is used to define the finite terms in $I_{1}$ and $I_{2}$, and we use

$$
\Gamma(\epsilon) I(-\epsilon, b, c)=b\left[\log \left(c / \Lambda_{\chi}^{2}\right)-2\right]-\sqrt{b^{2}-c} \log \left(\frac{b-\sqrt{b^{2}-c+i \epsilon}}{b+\sqrt{b^{2}-c+i \epsilon}}\right)
$$

where only finite pieces are shown. The matrix element for radiative decays can be written as

$$
\mathcal{M}=X \bar{B} S \cdot k T \cdot \epsilon_{\gamma} \quad+\quad Y \bar{B} S \cdot \epsilon_{\gamma} k \cdot T
$$


where $X$ and $Y$ include contributions from both one-loop graphs and the counterterm;

$$
\begin{aligned}
& X=-i e\left[Q_{T B} \frac{\Theta}{\Lambda_{\chi}}+\frac{1}{4 \pi^{2} f^{2}}\left(\beta_{T B B} I_{2}-\beta_{T T B}\left[I_{1}-\frac{2}{3} I_{2}\right]\right)\right] \\
& Y=-i e\left[-Q_{T B} \frac{\Theta}{\Lambda_{\chi}}+\frac{1}{4 \pi^{2} f^{2}}\left(\beta_{T B B} I_{1}-\beta_{T T B}\left[I_{2}-\frac{2}{3} I_{1}\right]\right)\right]
\end{aligned}
$$

where $\epsilon_{\gamma}$ is the photon polarization vector, $\beta_{T B B}$ and $\beta_{T T B}$ are the Clebsch-Gordan coefficients relevant for each decay (from one-loop graphs with an octet and decuplet baryon, respectively, in the intermediate state), and $Q_{T B}$ is the Clebsch-Gordan coefficient in the counterterm (see Appendix B). Note that the integrals $I_{1}$ and $I_{2}$ depend on $\omega_{\gamma}, \Delta m$, and $M$, and are therefore different for each intermediate state. The contribution from the one-loop wavefunction renormalisation graphs is not included, being higher order in the chiral expansion. Keeping explicit the difference between $f_{K}$ and $f_{\pi}$ in the computations, we use $1 / f_{K}^{2}$ for kaon loops and $1 / f_{\pi}^{2}$ for pion loops, where $f_{K}=1.22 f_{\pi}$. We find the width for a given radiative decay mode $\Gamma(T \rightarrow B \gamma)$ is

$$
\Gamma(T \rightarrow B \gamma)=\frac{\omega_{\gamma}^{3}}{8 \pi}\left[|X|^{2}+|Y|^{2}+\frac{2}{3}\left(X^{*} Y+Y^{*} X\right)\right]
$$

The $\Delta \rightarrow N \gamma$ branching ratio is believed to be $0.56-0.66 \%$ from studies of pion photoproduction. We use this to constrain the value of the counterterm $\Theta$ (remember that this counterterm is subtraction scheme dependent when determined to order $1 / \Lambda_{\chi}^{2}$ ). The width depends quadratically on $\Theta$, leading to two possible solutions. One of the solutions gives a branching ratio for $\Xi^{* 0} \rightarrow \Xi^{0} \gamma$ that is greater than the experimental upper limit of $4 \%$, which eliminates this as a physical solution. Therefore, all of the $\mathrm{SU}(3)$ allowed and $\mathrm{SU}(3)$ forbidden decay rates can be predicted as a function of $\mathcal{H}$. We found the range of allowed values for $\mathcal{H}$ in the previous section. We will here give a range for each decay rate which includes both experimental uncertainties on the widths of the resonances, and an estimate of the theoretical uncertainties (about 30\%) inherent in chiral perturbation theory. In computing the branching ratio for each decay we have used the following values for the widths (in $\mathrm{MeV}$ ) of the decuplet states [8]:

$$
\begin{aligned}
\Gamma\left(\Sigma^{* 0}\right)=36 \pm 5 & \Gamma\left(\Sigma^{*-}\right)=39.4 \pm 2.1 \\
\Gamma\left(\Sigma^{*+}\right)=35.8 \pm 0.8 & \Gamma\left(\Xi^{* 0}\right)=9.1 \pm 0.5 \\
\Gamma\left(\Xi^{*-}\right)=9.9 \pm 1.8 &
\end{aligned}
$$


The estimates for the branching fraction for each decay mode are given in Table 1. The ones in the lower box are $\mathrm{SU}(3)$ violating.

Table 1: Decuplet Branching Ratios (\%)

\begin{tabular}{|c|c|c|}
\hline Decay Mode & Branching Ratio (\%) & Experimental Limit (\%) \\
\hline$\Sigma^{*+} \rightarrow \Sigma^{+} \gamma$ & $0.2-0.6$ & $<5$ \\
$\Sigma^{* 0} \rightarrow \Sigma^{0} \gamma$ & $0.04-0.1$ & $<5$ \\
$\Sigma^{* 0} \rightarrow \Lambda \gamma$ & $0.8-1.3$ & $<5$ \\
$\Xi^{* 0} \rightarrow \Xi^{0} \gamma$ & $1.0-3.0$ & $<4$ \\
\hline$\Sigma^{*-} \rightarrow \Sigma^{-} \gamma$ & $0.004-0.006$ & $<5$ \\
$\Xi^{*-} \rightarrow \Xi^{-} \gamma$ & $0.01-0.03$ & $<4$ \\
\hline
\end{tabular}

The largest predicted branching ratio is for the $\Xi^{* 0}$, which is near the present experimental upper limit of $4 \%$. Similarly the branching ratio for $\Sigma^{* 0} \rightarrow \Lambda \gamma$ is close to the present bound. Although the SU(3) violating decays have much smaller branching ratios, a measurement of one or both of these branching fractions would give invaluable information on the nature of $\mathrm{SU}(3)$ violation in HBChPT. Further, it would also give an indication of whether our estimate derived from kaon loops is reliable. We expect that CEBAF will see these decay modes in the coming years; the measurement will be difficult, but the return will be enormous for its impact on our understanding of the role played by meson exchanges, and the consequent manifestation of QCD symmetries in these types of processes.

\section{Pole Graph Contribution to Nucleon Polarisability}

We are now in a position to comment on the polarisability of the nucleon which has been computed in chiral perturbation theory at order $1 / \Lambda_{\chi}^{2}$ with octet baryons as intermediate states [11] and with the inclusion of the decuplet of baryon resonances [12]. These calculations suffer from an uncertainty in how the decuplet pole graphs (fig. 5) contribute to this process. 1 The importance of including the decuplet of resonances in

1 We thank W. Broniowski for pointing out the potential importance of this graph to the magnetic susceptibility of the nucleon 
nucleon polarisability has been discussed in both the context of the Skyrme model and in chiral perturbation theory by Broniowski and Cohen [13. Now, the same graphs and vertices that appear in the radiative decay of the decuplet baryons are also in the pole graph contribution to the polarisability. Formally, we should only keep contributions at order $1 / \Lambda_{\chi}^{2}$ in the chiral expansion since contact terms at order $1 / \Lambda_{\chi}^{3}$ and higher are incalculable. To include the pole graph consistently, we must retain the loop corrected vertices in fig. 5 (each contributes a $1 / \Lambda_{\chi}$ and a $1 / \Lambda_{\chi}^{2}$ term) because the pole itself enhances the naive contribution of the graph by $\Lambda_{\chi} / \Delta m$; terms that appear, from vertex counting, to go as $1 / \Lambda_{\chi}^{3}$ are actually of order $1 / \Lambda_{\chi}^{2}$ and must be kept. In doing so, terms in the graph of order $1 / \Lambda_{\chi}^{3}$ (from naive vertex counting they are of order $1 / \Lambda_{\chi}^{4}$ ) are present, but since they are of lower order they are expected to be small. The pole graph with a $\Delta$ in the intermediate state does not contribute to the electric susceptibility, $\alpha$, but does give a finite contribution to the magnetic susceptibility, $\beta$. We find that

$$
\beta^{\text {pole }}=-\frac{1}{24 \pi \Delta m} \operatorname{Re}\left(X^{*} Y\right)
$$

where the integrals in $X$ and $Y$ are now evaluated at the off-shell point $\omega_{\gamma}=0$ (where $Y=-X)$. The allowed solution for the counterterm $\Theta$ indicates that the contribution from the pole graphs to $\beta$ is

$$
\beta^{\text {pole }}=(1-5) \times 10^{-4} \quad \mathrm{fm}^{3}
$$

This is consistent with the experimental determination of $\beta^{\text {expt }}=(3 \pm 2 \pm 2) \times 10^{-4} \mathrm{fm}^{3}$. It should be noted here that $\beta$ may be less well determined than the quoted experimental uncertainties suggest. The extraction of proton polarisabilities in these measurements use a result from dispersion relations, $2 \alpha_{p}+\beta_{p}=14.2 \times 10^{-4} \mathrm{fm}^{3}$ (ignoring a conservatively estimated uncertainty of $0.5 \times 10^{-4} \mathrm{fm}^{3}$ ), as input to a theoretical cross-section, and then fit $\alpha_{p}-\beta_{p}$ to their data [14] (for a review see ref. [15]). In principle though, both expressions can be extracted from their data, with the result that $\alpha_{p}$ does not change dramatically, but the uncertainty in $\beta_{p}$ completely overwhelms the quoted central value. For measurements of the neutron polarisabilities, it is only possible to extract $\alpha_{n}$ from the data, and the dispersion relation result of $\alpha_{n}+\beta_{n}=15.8 \pm 0.5 \times 10^{-4} \mathrm{fm}^{3}$ is then used to estimate $\beta_{n}$ [16]. What is now needed is an entirely experimental determination of both

2 We thank J. Schmiedmayer for pointing out the use of dispersion relations in the experimental analyses. 
$\alpha$ and $\beta$, independent of dispersion relations; these are the numbers that will allow us to better understand hadronic structure.

The large theoretical uncertainty comes from the approximations made in the $1 / M_{B}$, $1 / \Lambda_{\chi}$, and loop expansions. If we had naively used the vertices found from the radiative decay without evaluating at the off-shell point we would have found $\beta^{\text {pole }}=(4-8) \times$ $10^{-4} \mathrm{fm}^{3}$. This highlights the importance of retaining the momentum dependence of the photon vertex. Although the difference in the ranges given between the off-shell and the on-shell vertex calculations does not appear dramatic, in fact there is no overlap between the two. For instance, when the off-shell calculation gives $5 \times 10^{-4} \mathrm{fm}^{3}$, the corresponding value for the on-shell case is $8 \times 10^{-4} \mathrm{fm}^{3}$. The electric susceptibility appears to be dominated by long-distance pion loops, and the magnetic susceptibility by the $\Delta$ pole graph.

\section{Conclusions}

We computed the radiative decay rates of decuplet baryons. Two of the decay modes are forbidden in the limit of $\mathrm{SU}(3)$ but are induced at loop-level with the dominant contribution to the decay amplitude arising from kaon loops. We have computed the leading nonanalytic $\mathrm{SU}(3)$ violating contribution at order $m_{s}^{1 / 2}$ to all possible radiative decays of decuplet baryons arising at one-loop. The single (SU(3) symmetric) counterterm is determined by using the observed branching ratio for $\Delta \rightarrow N \gamma$ and the present upper limit on the branching ratio of $\Xi^{* 0} \rightarrow \Xi^{0} \gamma$. From these constraints we have made predictions of the branching ratios for $\Sigma^{*+} \rightarrow \Sigma^{+} \gamma, \Sigma^{* 0} \rightarrow \Sigma^{0} \gamma, \Sigma^{* 0} \rightarrow \Lambda \gamma, \Xi^{* 0} \rightarrow \Xi^{0} \gamma$, using the value of $\mathcal{H}$ found at one-loop. Branching ratios for the $\mathrm{SU}(3)$ violating decays $\Sigma^{*-} \rightarrow \Sigma^{-} \gamma$ and $\Xi^{*-} \rightarrow \Xi^{-} \gamma$ are independent of the counterterm. Some of these branching ratios should be observable in the near future, particularly $\Sigma^{*+} \rightarrow \Sigma^{+} \gamma, \Sigma^{* 0} \rightarrow \Lambda \gamma$ and $\Xi^{* 0} \rightarrow \Xi^{0} \gamma$, with the branching ratios predicted to be $\sim 1 \%$. It will be interesting to learn if these predictions are verified by experimental observation. In particular, if the branching ratios of the two decay modes which proceed only through $\mathrm{SU}(3)$ violation are found to be smaller than our predictions then this will be further evidence that the leading nonanalytic contribution from kaon loops overestimate the amount of $\mathrm{SU}(3)$ violation [7]. It is important that this is checked experimentally so we can determine if kaon loops are suppressed as suggested by Gasser and Leutwyler [17] in an approach they call improved chiral perturbation theory. 
We have also discussed the strong decays of the decuplet baryons. From comparison with experimental measurements, we have found that the bare value of the decuplet-octetmeson coupling constant, $\mathcal{C}$, lies in the interval $1.1<|\mathcal{C}|<1.3$, and that the decupletdecuplet-meson coupling constant, $\mathcal{H}$, lies in the interval $-2.8<\mathcal{H}<-1.6$. These couplings are in remarkable agreement with predictions based on an $\mathrm{SU}(6)$ spin-flavour symmetry between the heavy octet and decuplet baryon fields, if the goldstone bosons are assigned to a $\mathbf{3 5}$ representation. One might conclude from this that there is, in fact, an underlying $\mathrm{SU}(6)$ spin-flavour symmetry present in the hadronic sector.

Finally, we have estimated the $\Delta$ pole contribution to the polarisability of the nucleon including the momentum dependence of the photon vertex. This is consistent to order $1 / \Lambda_{\chi}^{2}$, dominates the magnetic susceptibility of the nucleon, and is compatible with current experimental data. We believe the calculations presented here provide another important probe in the study of the long-distance behavior of QCD.

MJS and MNB wish to thank the physics department at Duke University for their kind hospitality while some of this work was performed. We wish to thank E. Jenkins, A. Manohar and M. Luke for numerous discussions. MNB acknowledges the support of the Natural Science and Engineering Research Council (NSERC) of Canada. MJS acknowledges the support of a Superconducting Supercollider National Fellowship from the Texas National Research Laboratory Commission under grant FCFY9219. RPS acknowledges the support of DOE grant DE-FG05-90ER40592. 


\section{Appendix A}

A.1 Clebsch-Gordan coefficients for the 1PI contributions to the renormalisation of $\mathcal{C}$.

$$
\begin{aligned}
& \alpha_{\Delta N}=\frac{1}{3}-\frac{10}{81} \mathcal{H}^{2}+\frac{20}{9} F \mathcal{H}-\frac{10}{27} D \mathcal{H}-\frac{1}{18} \mathcal{C}^{2}-\frac{2}{3} D^{2}-4 D F+2 F^{2} \\
& \alpha_{\Sigma^{*} \Lambda}=\frac{1}{3}-\frac{20}{81} \mathcal{H}^{2}+\frac{10}{27} D \mathcal{H}+\frac{10}{3} F \mathcal{H}-\frac{1}{9} \mathcal{C}^{2}-\frac{16}{9} D^{2}-\frac{16}{3} D F \\
& \alpha_{\Sigma^{*} \Sigma}=\frac{1}{3}-\frac{80}{81} \mathcal{H}^{2}+\frac{20}{9} F \mathcal{H}-\frac{1}{3} \mathcal{C}^{2}-4 D^{2}+4 F^{2}-\frac{16}{3} F D \\
& \alpha_{\Xi^{*} \Xi}=\frac{1}{3}-\frac{20}{27} \mathcal{H}^{2}+\frac{25}{27} D \mathcal{H}+\frac{35}{9} F \mathcal{H}-\frac{5}{18} \mathcal{C}^{2}-4 D^{2}-\frac{20}{3} D F
\end{aligned}
$$

A.2 Clebsch-Gordan coefficients for the wavefunction renormalisation of the decuplet and octet baryons.

$$
\begin{array}{ll}
\lambda_{\Delta}=\frac{25}{27} \mathcal{H}^{2}+\mathcal{C}^{2} & \lambda_{N}=\mathcal{C}^{2}+\frac{17}{3} D^{2}+15 F^{2}-10 D F \\
\lambda_{\Sigma^{*}}=\frac{40}{27} \mathcal{H}^{2}+\frac{4}{3} \mathcal{C}^{2} & \lambda_{\Lambda}=2 \mathcal{C}^{2}+\frac{14}{3} D^{2}+18 F^{2} \\
& \lambda_{\Sigma}=\frac{14}{3} C^{2}+\frac{26}{3} D^{2}+6 F^{2} \\
\lambda_{\Xi^{*}}=\frac{55}{27} \mathcal{H}^{2}+\frac{5}{3} \mathcal{C}^{2} & \lambda_{\Xi}=\frac{13}{3} C^{2}+\frac{17}{3} D^{2}+15 F^{2}+10 F D
\end{array}
$$




\section{Appendix B}

B.1 Clebsch-Gordan coefficients for radiative decays of the decuplet.

$\Delta^{+} \rightarrow p \gamma$

$$
\begin{aligned}
& \beta_{\Delta+\Delta^{0} p}=\frac{2}{3 \sqrt{3}} \mathcal{C H} \\
& \beta_{\Delta+\Sigma^{0} p}=-\frac{1}{\sqrt{3}} \mathcal{C}(D-F) \\
& \Xi^{*-} \rightarrow \Xi^{-} \gamma \\
& \beta_{\Xi^{*-} \Xi^{0} \Xi^{-}}=\frac{1}{\sqrt{3}} \mathcal{C}(D-F) \quad \beta_{\Xi^{*-} \Lambda \Xi^{-}}=-\frac{1}{2 \sqrt{3}} \mathcal{C}(D-3 F) \\
& \beta_{\Sigma^{*-} \Delta^{0} \Sigma^{-}}=\frac{1}{3 \sqrt{3}} \mathcal{C H} \\
& \beta_{\Xi^{* 0} \Omega^{-} \Xi^{0}}=-\frac{1}{\sqrt{3}} \mathcal{C H} \\
& \beta_{\Xi^{*-} \Xi^{* 0} \Xi^{-}}=-\frac{1}{3 \sqrt{3}} \mathcal{C H} \\
& \beta_{\Xi^{*-} \Sigma^{0} \Xi^{-}}=-\frac{1}{2 \sqrt{3}} \mathcal{C}(D+F)
\end{aligned}
$$


B.2 Clebsch-Gordan coefficients for contribution to the radiative decay from the counterterm.
$Q_{\Delta^{+} p}=\frac{1}{\sqrt{3}}$
$Q_{\Delta^{0} n}=\frac{1}{\sqrt{3}}$
$Q_{\Sigma^{* 0} \Lambda}=-\frac{1}{2}$
$Q_{\Sigma^{* 0} \Sigma^{0}}=\frac{1}{\sqrt{12}}$
$Q_{\Sigma^{*+} \Sigma^{+}}=-\frac{1}{\sqrt{3}}$
$Q_{\Sigma^{*-} \Sigma^{-}}=0$
$Q_{\Xi^{* 0} \Xi^{0}}=-\frac{1}{\sqrt{3}}$
$Q_{\Xi^{*-} \Xi^{-}}=0$ 


\section{References}

[1] E. Jenkins and A. Manohar, Phys. Lett. 255B (1991) 558 ; Phys. Lett. 259B (1991) 353.

[2] M.B. Wise, "New Symmetries of the Strong Interaction," lectures presented at the Lake Louise Winter Institute, Feb. 1991

[3] E. Jenkins and A. Manohar, Proceedings of the workshop on "Effective Field Theories of the Standard Model ", ed. U. Meissner, World Scientific (1992)

[4] C. Carone and H. Georgi, Nucl. Phys. B375 (1992) 243

[5] S. Coleman and S.L. Glashow, Phys. Rev. Lett. 6 (1961) 423.

[6] D.G. Caldi and H. Pagels, Phys. Rev. D10 (1974) 3739

[7] E. Jenkins, M. Luke, A.V. Manohar and M.J. Savage, UCSD/PTH-92-34

[8] Particle Data Group, Phys. Rev. D45 (1992) 1

[9] E. Kaxiras, E.J. Moniz and M. Soyeur, Phys. Rev. D32 (1985) 695

[10] H. Lipkin, Phys. Rev. D7 (1973) 846

[11] V. Bernard et al, Preprint BUTP-92/15, CRN 92-24 (1992)

[12] M.N. Butler and M.J. Savage, UCSD/PTH 92-30, QUSTH 92-04 (1992)

[13] W. Broniowski and T.D. Cohen, University of Maryland Preprint U.of.MD PP 92-193; T.D. Cohen and W. Broniowski, University of Maryland Preprint U.of.MD PP 92-191.

[14] F.J. Fiederspiel et al., Phys. Rev. Lett. 67 (1991) 1511

[15] A.I. L'vov, talk presented at Brookhaven Workshop on Hadron Structure from Photoreactions at Intermediate Energies, May 1992.

[16] J. Schmiedmayer et al., Phys. Rev. Lett. 66 (1991) 1015

[17] J. Gasser and H. Leutwyler, Phys. Rep. 87 (1982) 77. 


\section{Figure Captions}

Fig. 1. 1PI graphs for the strong decay of a decuplet baryon to an octet baryon.

Fig. 2. Graphs contributing to wavefunction renormalisation of the decuplet and octet baryons.

Fig. 3. The decuplet-octet-meson coupling constant $\mathcal{C}$ as a function of the decupletdecuplet-meson coupling constant $\mathcal{H}$ for the decays $\Delta^{++} \rightarrow p \pi^{+}, \Sigma^{*+} \rightarrow$ $\Sigma^{0} \pi^{+}, \Sigma^{*+} \rightarrow \Lambda \pi^{+}$and $\Xi^{* 0} \rightarrow \Xi^{-} \pi^{+}$. The width of each line represents the $1 \sigma$ error arising from experimental determinations of the baryon resonant width. There is no theoretical uncertainty included in the curves. Note that the $\operatorname{sign}$ of $\mathcal{C}$ is chosen for ease of comparison to $\mathrm{SU}(6)$ predictions, but is in fact undetermined in HBChPT.

Fig. 4. Graphs contributing to the radiative decay of decuplet baryons. The heavy line represents a decuplet baryon, the solid line an octet baryon, the dashed line an octet meson, and the wavy line a photon. We work in $\epsilon_{\gamma} \cdot v=0$ gauge where there is no direct coupling of the photon to either the octet or decuplet baryon.

Fig. 5. Pole graph contribution to the polarisability of the nucleon from decuplet intermediate states. 